\title{
CRYPTOCOCCAL MENINGITIS IN A NON-AIDS PATIENT
}

\author{
Khanal $\mathbf{B}^{1}$, Sharma S K $\mathrm{K}^{1}$, Deb $\mathbf{M}^{1}$
}

ABSTRACT

Cryptococcal meningitis that occurred in a patient with no obvious predisposing factor is reported. The necessity to identify this entity in the cases of chronic meningitis is discussed.

\section{Key Words: Meningitis, Cryptococcus neoformans.}

\section{INTRODUCTION}

Cryptococcosis is an infection caused by an encapsulated yeast Cryptococcus neoformans, considered to be rare prior to Acquired Immune Deficiency Syndrome (AIDS) epidemic. Although AIDS is now the major predisposing factor for cryptococcosis, this condition is also reported in non - AIDS group which includes a large percentage of patients with deficiencies in cell mediated immunity and some others without any obvious predisposing factor. ${ }^{1-3}$

Any organ or tissue is subject to invasion by this saprophytic fungus after it enters the host by inhalation. However, due to its neurotropism, meningitis is the commonly encountered manifestation of cyptococcosis. ${ }^{2,3}$ Subacute onset, indolent course and the nonspecific presentations of the disease resemble tuberculous meningitis. ${ }^{2,3}$ We report a case of cryptococcal meningitis that occurred in a Human Immunodeficiency Virus (HIV) negative patient who was initially treated for tuberculous meningitis due to similar clinical features.

\section{CASE REPORT}

A 35 years male, businessman, resident of Jhapa in Eastern Nepal presented to the Emergency of B P Koirala Institute of Health Sciences Hospital, with 22 days history of high grade fever, headache followed by altered

1. B. P. Koirala Institute of Health Sciences, Dharan, Nepal.

Address for correspondence : Dr. Basudha Khanal

Department of Microbiology

B. P. Koirala Institute of Health Sciences, Dharan, Nepal

Phone: 00977-25-25555, Ext.: 2614, Fax: 977-25-20251

Email: basudhak@hotmail.com, basudhak@yahoo.com 
sensorium off and on. He reported to a local hospital where he was started on antituberculous therapy (ATT) and steroid for the same complaints. Improvement was not observed and the patient reported to this hospital after one week. His past history and family history were not contributory.

On examination he was drowsy, neck rigidity and Kerning sign were present. Other examination did not reveal any abnormality. X-ray chest taken a week earlier showed bilateral apical opacity. In view of these findings, provisional diagnosis of TB meningitis was made and the ATT was continued.

Post admission a few laboratory investigations were done to obtain baseline data. Hemogram, blood sugar and renal profile were within normal limits. Lumbar puncture was done,

Fig. 1

Fig. 1, India Ink preparation of cerebrospinal fluid showing capsulated and budding morphology of Crytococcus neoformans ( $X 1000$ ).
Cerebrospinal fluid (CSF) was clear. There were no cells but high protein $(108 \mathrm{mg} / \mathrm{dl})$ and less glucose $(24 \mathrm{mg} / \mathrm{dl})$ were a significant findings. Ziehl Neelsen stained smear did not show any acid fast bacilli. Gram variable structures surrounded by a wide halo but no inflammatory cells observed in grams staining led to the suspicion of cryptococcal infection. India Ink preparation of centrifuged deposits of CSF revealed multiple, capsulated, budding yeast cells characteristic of $C$. neoformans (Figure). On this finding diagnosis of cryptococcal meningitis was made and treatment was started with Amphotericin B. Screening Enzyme linked immunosorbent assay (ELISA) of his serum was negative for HIV antibody. Due to lack of facilities other parameters for a deficient cell mediated immunity, could not be assessed. CSF culture for pyogenic meningitis and tuberculosis were sterile. Culture of CSF on Sabouraud's Dextrose Agar (SDA) yielded creamy white colony of budding yeast cells after 48 hours of incubation. C. neoformans was diagnosed on the basis of characteristic morphology, growth at $37^{\circ} \mathrm{C}$ and biochemical reactions ${ }^{3}$.

The patient improved clinically. CSF microscopy and culture repeated after 7 days were sterile. However, patient left against medical advice before the therapy could be completed.

\section{DISCUSSION}

The dramatic increase in the number of cases of cryptococcosis in various parts of the world is attributed to the ever increasing population of immunocompromised host as a result of chemotherapy and organ transplantation and disease like AIDS. Awareness among clinicians and microbiologist of this infection have also 
contributed significantly to the diagnosis in recent time. ${ }^{3}$ C. neoformans can infect skin, bone, and other visceral organs but has predilection for the central nervous system. Bilateral opacity in the apices of the lungs of this patient is suggestive of a primary pulmonary infection which was possibly asymptomatic.

The diagnosis of cryptococcal meningitis can be very challenging since onset of symptom is usually subacute or chronic and runs an indolent course resembling several other conditions including TB meningitis which is common in this part of the world. Although, cryptococcal meningitis is reported from various parts of the world both in immunocompromised and immunocompetent individuals, to the best of our knowledge this is the first reported case of cryptococcal meningitis in Nepal. ${ }^{1,46}$ For the effective management of such cases the diagnosis is most crucial which is based on a high index of suspicion both in immunocompromised and immunocompetent individuals, presenting with subacute or chronic meningitis. Prompt diagnosis and immediate institution of the antifungal therapy are essential to prevent the morbidity and mortality caused by this illness.

It is therefore concluded that a differential diagnosis of cryptococcal meningitis should be always kept in mind in all cases of subacute and chronic meningitis. Simple microbiology technique of India Ink preparation and direct microscopy could help to arrive at a rapid diagnosis and immensely help the clinician in the management.

\section{REFERENCES}

1. Mitchell TG, Perfect J R Cryptococcosis in the era of AIDS - 100 years after the discovery of Cryptococcus neoformans. Clin Microbiol Rev. 1995; 8:515-548.

2. Diamond RD. Cryptococcus neoformans, In: Mandell GT, Bennett JE, Dolin R, eds. Mandell, Doughlas and Bennett's Principles and Practice of Infectious diseases, vol. 2, Philadelphia, Churchill Livingstone, 2000: 2707 - 2718.

3. Cox GM, Perfect JR. Cryptococcus neoformans var neoformans and gattii and Trichosporon species, In: Ajello L, Hay RJ, eds. Topley and Wilson's Microbiology and Microbial infection, vol 4, London, Arnold, 1998: 461 - 484.

4. Sanchetee PC. Cryptococcal meningitis in immunocompetent host, J Assos Phys India 1998; 46:617-619.

5. Madan M, Ranjitham M, Chandrasekaran S, Sudhakar, Cryptococceal meningitis in Immunocompetent Individuals. J Assos Phys India 1999; 47:933-934.

6. Chuck SL, Saunde MA. Infection with Cryptococcus neoformans in the acquired immunodeficiency syndrome, N Engl J Med 1989; 321:794-799. 